\title{
Explicit Multistep Mixed Finite Element Method for RLW Equation
}

\author{
Yang Liu, ${ }^{1}$ Hong Li, ${ }^{1}$ Yanwei Du, ${ }^{1}$ and Jinfeng Wang ${ }^{2}$ \\ ${ }^{1}$ School of Mathematical Sciences, Inner Mongolia University, Hohhot 010021, China \\ ${ }^{2}$ School of Statistics and Mathematics, Inner Mongolia University of Finance and Economics, Hohhot 010070, China \\ Correspondence should be addressed to Yang Liu; mathliuyang@aliyun.com and Hong Li; smslh@imu.edu.cn
}

Received 15 February 2013; Revised 22 April 2013; Accepted 30 April 2013

Academic Editor: Marco Donatelli

Copyright (c) 2013 Yang Liu et al. This is an open access article distributed under the Creative Commons Attribution License, which permits unrestricted use, distribution, and reproduction in any medium, provided the original work is properly cited.

An explicit multistep mixed finite element method is proposed and discussed for regularized long wave (RLW) equation. The spatial direction is approximated by the mixed Galerkin method using mixed linear space finite elements, and the time direction is discretized by the explicit multistep method. The optimal error estimates in $L^{2}$ and $H^{1}$ norms for the scalar unknown $u$ and its flux $q=u_{x}$ based on time explicit multistep method are derived. Some numerical results are given to verify our theoretical analysis and illustrate the efficiency of our method.

\section{Introduction}

In this paper, we consider the following initial boundary problem of RLW equation:

$$
\begin{gathered}
u_{t}+\gamma u_{x}+\delta u u_{x}-\beta u_{x x t}=0, \quad(x, t) \in I \times J, \\
u(a, t)=u(b, t)=0, \quad t \in \bar{J}, \\
u(x, 0)=u_{0}(x), \quad x \in \bar{I},
\end{gathered}
$$

where $I=(a, b)$ is a bounded open interval, $J=(0, T]$ with $0<T<\infty$. The initial value $u_{0}(x)$ is given function, and the coefficients $\beta, \gamma, \delta$ are all positive constants.

In recent years, large nonlinear phenomena are found in many research fields, for example, physics, biology, fluid dynamics, and so forth. These phenomena can be described by the mathematical model of some nonlinear evolution equations. In particular, some attention has also been paid to nonlinear RLW equations $[1,2]$ which play a very important role in the study of nonlinear dispersive waves. Solitary waves are wave packet or pulses, which propagate in nonlinear dispersive media. Due to dynamical balance between the nonlinear and dispersive effects, these waves retain an unchanged waveform. A soliton is a very special type of solitary wave, which also keeps its waveform after collision with other solitons. The regularized long wave
(RLW) equation is an alternative description of nonlinear dispersive waves to the more usual Korteweg-de Vries (KdV) equation. Mathematical theories and numerical methods for (1) were considered in [1-24]. The existence and uniqueness of the solution of RLW equation are discussed in [5]. Their analytical solutions were found under restricted initial and boundary conditions, and therefore they got interest from a numerical point of view. Several numerical methods for the solution of the RLW equation have been introduced in the literature. These include a variety of difference methods [5-8, 17], finite element methods based on Galerkin and collocation principles [9-13], mixed finite element methods [14-16], meshfree method [18], adomian decomposition method [19], and so on.

In [25], Chatzipantelidis studied the explicit multistep methods for some nonlinear partial differential equations and discussed some mathematical theories. Akrivis et al. [26] studied the multistep method for some nonlinear evolution equations. Mei and Chen [20] presented the explicit multistep method based on Galerkin method for regularized long wave (RLW) equation. In this paper, our purpose is to propose and study an explicit multistep mixed method, which combines a mixed Galerkin method in the spatial direction and the explicit multistep method in the time direction, for RLW equation. We derive optimal error estimates in $L^{2}$ and $H^{1}$ norms for the scalar unknown $u$ and its flux $q=u_{x}$ 
for the fully discrete explicit multistep mixed scheme and compare our method's accuracy with some other numerical schemes. Compared to the numerical methods in $[20,25,26]$, we not only obtain the approximation solution for $u$, but also get the approximation solution for $q=u_{x}$.

The layout of the paper is as follows. In Section 2, an explicit multistep mixed scheme and numerical process are given. The optimal error estimates in $L^{2}$ and $H^{1}$ norms for the scalar unknown $u$ and its flux $q=u_{x}$ for the fully discrete explicit multistep mixed scheme are proved in Section 3. In Section 4, some numerical results are shown to confirm our theoretical analysis. Finally, some concluding remarks are given in Section 5. Throughout this paper, $C$ will denote a generic positive constant which does not depend on the spatial mesh parameter $h$ or time discretization parameter $\Delta t$.

\section{The Mixed Numerical Scheme}

With the auxiliary variable $q=u_{x}$, we reformulate (1) as the following first-order coupled system:

$$
\begin{gathered}
u_{x}=q \\
u_{t}+\gamma q+\delta u q-\beta q_{x t}=0 .
\end{gathered}
$$

We consider the following mixed weak formulation of (2). Find $\{u, q\}:[0, T] \mapsto H_{0}^{1} \times H^{1}$ satisfying:

$$
\begin{gathered}
\left(u_{x}, v_{x}\right)=\left(q, v_{x}\right), \quad \forall v \in H_{0}^{1}, \\
\left(q_{t}, w\right)+\beta\left(q_{x t}, w_{x}\right)=\gamma\left(q, w_{x}\right)+\delta\left(u q, w_{x}\right), \quad \forall w \in H^{1} .
\end{gathered}
$$

Noting the Dirichlet boundary conditions $u_{t}(a, t)=u_{t}(b, t)=$ 0 and $q_{t}=u_{x t}$, we can get $\left(u_{t},-w_{x}\right)=\left(u_{x t}, w\right)=\left(q_{t}, w\right)$ easily and then get the scheme (4).

Let $V_{h}$ and $W_{h}$ be finite dimensional subspaces of $H_{0}^{1}$ and $H^{1}$, respectively, defined by

$$
\begin{gathered}
V_{h}=\left\{v_{h}\left|v_{h} \in C^{0}(\bar{I}), v_{h}\right|_{I_{j}} \in P_{k}\left(I_{j}\right),\right. \\
\left.\forall I_{j} \in T_{h}, v_{h}(a)=v_{h}(b)=0\right\} \\
\subset H_{0}^{1}, \\
W_{h}=\left\{w_{h}\left|w_{h} \in C^{0}(\bar{I}), w_{h}\right|_{I_{j}} \in P_{r}\left(I_{j}\right), \forall I_{j} \in T_{h}\right\} \subset H^{1},
\end{gathered}
$$

where $T_{h}$ is a partition of $\bar{I}=[a, b]$ into $N$ subintervals $I_{j}=\left[x_{j}, x_{j+1}\right], j=0,1,2, \ldots,(N-1), h_{j}=h_{j+1}-h_{j}$, $h=\max _{0 \leq j \leq N-1} h_{j}$, and $P_{m}\left(I_{j}\right)$ denotes the polynomials of degree less than or equal to $m$ in $I_{j}$.
The semidiscrete mixed finite element method for (3) and (4) consists in determining $\left\{u^{h}, q^{h}\right\}:[0, T] \mapsto V_{h} \times W_{h}$ such that

$$
\begin{array}{r}
\left(u_{x}^{h}, v_{x}^{h}\right)=\left(q^{h}, v_{x}^{h}\right), \quad \forall v^{h} \in V_{h}, \\
\left(q_{t}^{h}, w^{h}\right)+\beta\left(q_{x t}^{h}, w_{x}^{h}\right)=\gamma\left(q^{h}, w_{x}^{h}\right)+\delta\left(u^{h} q^{h}, w_{x}^{h}\right), \\
\forall w^{h} \in W_{h} .
\end{array}
$$

In the following discussion, we will give an explicit multistep mixed scheme. We take linear finite element spaces $V_{h}=\operatorname{span}\left\{\varphi_{0}, \varphi_{1}, \ldots, \varphi_{N}\right\}$ and $W_{h}=\operatorname{span}\left\{\varphi_{0}, \varphi_{1}, \ldots, \varphi_{N}\right\}$, and then $u_{h}$ and $q_{h}$ can be expressed as the following formulation:

$$
\begin{aligned}
& u_{h}(x, t)=\sum_{i=0}^{N} u_{i}(t) \varphi_{i}(x), \quad(x, t) \in \Omega \times J, \\
& q_{h}(x, t)=\sum_{i=0}^{N} q_{i}(t) \varphi_{i}(x), \quad(x, t) \in \Omega \times J .
\end{aligned}
$$

Substitute (8) into (6) and (7), and take $v^{h}=\varphi_{j}$ and $w^{h}=\varphi_{j}$ in (6) and (7), respectively, to obtain

$$
\begin{aligned}
& \sum_{i=0}^{N}\left[\left(\int_{a}^{b} \varphi_{i} \cdot \varphi_{j} d x+\beta \int_{a}^{b} \varphi_{i x} \cdot \varphi_{j x} d x\right) \frac{\partial q_{i}}{\partial t}\right. \\
& \left.\quad-\left(\gamma \int_{a}^{b} \varphi_{i} \cdot \varphi_{j x} d x+\delta \int_{a}^{b}\left(\sum_{i=0}^{N} u_{i} \varphi_{i}\right) \varphi_{i} \cdot \varphi_{j x} d x\right) q_{i}\right] \\
& \quad=0 \\
& \sum_{i=0}^{N}\left[\left(\int_{a}^{b} \varphi_{i x} \cdot \varphi_{j x} d x\right) u_{i}-\left(\int_{a}^{b} \varphi_{i} \cdot \varphi_{j x} d x\right) q_{i}\right]=0,
\end{aligned}
$$

where $j=0,1,2, \ldots, N$.

We subdivide the space variable domain $[a, b]$ into uniform subintervals with $N+1$ grid points $x_{k}, k=0, \ldots, N$, such that $a=x_{0}<x_{1}<\cdots<x_{N}=b, h=x_{k+1}-x_{k}=$ $(b-a) / N$. Using the local coordinate transformation $x=$ $x_{k}+\mu h, 0 \leq \mu \leq 1$, we transform a subinterval $\left[x_{k}, x_{k+1}\right]$ into a standard interval $[0,1]$. Furthemore, we have

$$
\begin{aligned}
\sum_{i=k}^{k+1} & {\left[\left(\int_{0}^{1} \varphi_{i} \cdot \varphi_{j} d x+\frac{\beta}{h^{2}} \int_{0}^{1} \varphi_{i x} \cdot \varphi_{j x} d x\right) \frac{\partial q_{i}}{\partial t}\right.} \\
& \left.-\frac{1}{h}\left(\gamma \int_{0}^{1} \varphi_{i} \cdot \varphi_{j x} d x+\delta \int_{0}^{1}\left(\sum_{i=0}^{N} u_{i} \varphi_{i}\right) \varphi_{i} \cdot \varphi_{j x} d x\right) q_{i}\right] \\
& =0, \\
\sum_{i=k}^{k+1}[ & \left.\frac{1}{h^{2}}\left(\int_{0}^{1} \varphi_{i x} \cdot \varphi_{j x} d x\right) u_{i}-\frac{1}{h}\left(\int_{a}^{b} \varphi_{i} \cdot \varphi_{j x} d x\right) q_{i}\right]=0 .
\end{aligned}
$$


TABLE 1: Solitary wave Amp. 0.3 and the errors in $L^{2}$ and $L^{\infty}$ norms for $u, Q_{1}, Q_{2}$, and $Q_{3}$ at $t=20, h=0.125, \Delta t=0.1$, and $-40 \leq x \leq 60$.

\begin{tabular}{lcccccc}
\hline Method & Time & $Q_{1}$ & $Q_{2}$ & $Q_{3}$ & $L^{2}$ for $u$ & 0 \\
& 0 & 3.9797 & 0.8104 & 2.5787 & $L^{\infty}$ for $u$ \\
\hline \multirow{3}{*}{ Our method } & 4 & 3.9797 & 0.8104 & 2.5786 & $3.6304 e-004$ & $5.2892 e-005$ \\
& 8 & 3.9797 & 0.8104 & 2.5786 & $7.2873 e-004$ & $5.8664 e-005$ \\
& 12 & 3.9797 & 0.8104 & 2.5787 & $1.0817 e-003$ & $6.3283 e-005$ \\
& 16 & 3.9795 & 0.8104 & 2.5787 & $1.4186 e-003$ & $6.8001 e-005$ \\
{$[20]$} & 20 & 3.9790 & 0.8103 & 2.5785 & $1.7396 e-003$ & $7.7154 e-005$ \\
{$[21]$} & 20 & 3.9800 & 0.8104 & 2.5792 & $1.7569 e-003$ & $6.8432 e-004$ \\
{$[22]$} & 20 & 3.9820 & 0.8087 & 2.5730 & $4.688 e-003$ & $1.755 e-003$ \\
{$[23]$} & 20 & 3.9905 & 0.8235 & 2.6740 & $2.157 e-003$ & - \\
{$[24]$} & 20 & 3.9616 & 0.8042 & 2.5583 & $0.018 e-003$ & $1.566 e-003$ \\
\hline
\end{tabular}

TABLE 2: Convergence order and error in $L^{2}$ norm for $u$ of time with $h=0.125$ and $c=0.1$.

\begin{tabular}{lccccc}
\hline Time & $\Delta t=0.4$ & $\Delta t=0.2$ & $\Delta t=0.1$ & Order $(0.2 / 0.4)$ & Order $(0.1 / 0.2)$ \\
\hline 4 & $5.3805 e-003$ & $1.4267 e-003$ & $3.6304 e-004$ & 1.9151 & 1.9906 \\
2.0145 \\
8 & $1.1688 e-002$ & $2.9411 e-003$ & $7.2873 e-004$ & 2.0188 & 2.0241 \\
12 & $1.7830 e-002$ & $4.3997 e-003$ & $1.0817 e-003$ & 2.0360 & 2.0295 \\
16 & $2.3751 e-002$ & $5.7916 e-003$ & $1.4186 e-003$ & 2.0486 & 2.0321 \\
20 & $2.9434 e-002$ & $7.1148 e-003$ & $1.7396 e-003$ & &
\end{tabular}

We take linear basis functions defined as follows:

$$
L_{1}=1-\mu, \quad L_{2}=\mu,
$$

and then the variables $u$ and $q$ over the element $\left[x_{k}, x_{k+1}\right]$ are written as

$$
u^{e}=\sum_{j=1}^{2} L_{j} u_{j}, \quad q^{e}=\sum_{j=1}^{2} L_{j} q_{j} .
$$

Then, we get the following equations:

$$
\begin{aligned}
& \sum_{i=1}^{2}\left[\left(\int_{0}^{1} L_{i} \cdot L_{j} d \mu+\frac{\beta}{h^{2}} \int_{0}^{1} L_{i \mu} \cdot L_{j \mu} d \mu\right) \frac{\partial q_{i}}{\partial t}\right. \\
& \left.\quad-\frac{1}{h}\left(\gamma \int_{0}^{1} L_{i} \cdot L_{j \mu} d \mu+\delta \int_{0}^{1}\left(\sum_{i=1}^{2} u_{i} L_{i}\right) L_{i} \cdot L_{j \mu} d \mu\right) q_{i}\right] \\
& \quad=0, \\
& \sum_{i=1}^{2}\left[\frac{1}{h^{2}}\left(\int_{0}^{1} L_{i \mu} \cdot L_{j \mu} d \mu\right) u_{i}-\frac{1}{h}\left(\int_{a}^{b} L_{i} \cdot L_{j \mu} d \mu\right) q_{i}\right]=0 .
\end{aligned}
$$

Then, the system (13) has the following matrix form:

$$
\begin{gathered}
\left(A_{i j}^{e}+\beta B_{i j}^{e}\right) \frac{\partial \mathbf{q}^{e}}{\partial t}-\left(\gamma C_{i j}^{e}+\delta D_{i j}^{e}\left(u^{e}\right)\right) \mathbf{q}^{e}=0, \\
B_{i j}^{e} \mathbf{u}^{e}-C_{i j}^{e} \mathbf{q}^{e}=0
\end{gathered}
$$

with the following element matrices:

$$
\begin{gathered}
\mathbf{u}^{e}=\left(u_{1}, u_{2}\right)^{T}, \quad \mathbf{q}^{e}=\left(q_{1}, q_{2}\right)^{T}, \\
A_{i j}^{e}=\int_{0}^{1} L_{i} \cdot L_{j} d \mu, \quad B_{i j}^{e}=\frac{1}{h^{2}} \int_{0}^{1} L_{i \mu} \cdot L_{j \mu} d \mu, \\
C_{i j}^{e}=\frac{1}{h} \int_{0}^{1} L_{i} \cdot L_{j \mu} d \mu, \\
D_{j k}^{e}=\frac{\delta}{h} \int_{0}^{1}\left(\sum_{i=1}^{2} u_{i} L_{i}\right) L_{i \mu} \cdot L_{j} d \mu .
\end{gathered}
$$

Assembling contributions from all elements, we obtain the following coupled system of nonlinear matrix equations:

$$
\begin{gathered}
(A+\beta B) \frac{\partial \mathbf{q}_{h}}{\partial t}-\left(\gamma C+\delta D\left(\mathbf{u}_{h}\right)\right) \mathbf{q}_{h}=0, \\
B \mathbf{u}_{h}-C \mathbf{q}_{h}=0 .
\end{gathered}
$$

To formulate a fully discrete scheme, we consider a uniform partition of $\bar{J}=[0, T]$ with time step length $\Delta t=T / N$, $N \in \mathbf{Z}^{+}$, and time levels $t^{n}=n \Delta t, n=0, \ldots, N$. We now discuss a fully discrete scheme based on a linear explicit multistep method. We now define $U^{n} \in V_{h}$ and $Z^{n} \in W_{h}$ as approximations to $u\left(t^{n}\right)$ and $q\left(t_{n}\right)$, respectively, and formulate the following fully discrete linear explicit multistep mixed scheme:

$$
\begin{gathered}
(A+\beta B) \sum_{i=0}^{p} \alpha_{i} Z^{n+i}=\Delta t \sum_{i=0}^{p-1} \sigma_{i}\left[\left(\gamma C+\delta D\left(U^{n+i}\right)\right) Z^{n+i}\right], \\
B U^{n+p}=C Z^{n+p},
\end{gathered}
$$


TABLE 3: Convergence order and error in $L^{\infty}$ norm for $u$ of time with $h=0.125$ and $c=0.1$.

\begin{tabular}{lccccc}
\hline Time & $\Delta t=0.4$ & $\Delta t=0.2$ & $\Delta t=0.1$ & Order $(0.2 / 0.4)$ & Order $(0.1 / 0.2)$ \\
\hline 4 & $8.0743 e-004$ & $2.0299 e-004$ & $5.2892 e-005$ & 1.9919 & 1.9403 \\
8 & $9.1778 e-004$ & $2.2908 e-004$ & $5.8664 e-005$ & 2.0023 & 1.9653 \\
12 & $9.9866 e-004$ & $2.4966 e-004$ & $6.3283 e-005$ & 2.0000 & 1.9801 \\
16 & $1.0718 e-003$ & $2.6685 e-004$ & $6.8001 e-005$ & 2.0059 & 1.9724 \\
20 & $1.1277 e-003$ & $2.8609 e-004$ & $7.7154 e-005$ & 1.9788 & 1.8907 \\
\hline
\end{tabular}

TABLE 4: Convergence order and error in $L^{2}$ norm for $q$ of time with $h=0.125$ and $c=0.1$.

\begin{tabular}{lccccc}
\hline Time & $\Delta t=0.4$ & $\Delta t=0.2$ & $\Delta t=0.1$ & Order $(0.2 / 0.4)$ & Order $(0.1 / 0.2)$ \\
\hline 4 & $2.4430 e-003$ & $6.4427 e-004$ & $1.6260 e-004$ & 1.9229 & 1.9863 \\
8 & $5.1823 e-003$ & $1.2934 e-003$ & $3.1976 e-004$ & 2.0024 & 2.0161 \\
12 & $7.6616 e-003$ & $1.8713 e-003$ & $4.5963 e-004$ & 2.0336 & 2.0255 \\
16 & $9.8719 e-003$ & $2.3787 e-003$ & $5.8232 e-004$ & 2.0532 & 2.0303 \\
20 & $1.1843 e-002$ & $2.8251 e-003$ & $6.8991 e-004$ & 2.0677 & 2.0338 \\
\hline
\end{tabular}

with given the initial approximations $U^{0}, \ldots, U^{p-1}$ and $Z^{0}, \ldots, Z^{p-1}$. In the explicit multistep mixed system (17), the parameter variable $\alpha_{i}$ and $\sigma_{i}$ is described by the coefficients of the term $\chi^{i}$, for the following polynomials $\alpha(\chi)$ and $\sigma(\chi)$, respectively:

$$
\begin{gathered}
\alpha(\chi):=\sum_{j=1}^{p} \frac{1}{j} \chi^{p-j}(\chi-1)^{j}, \\
\sigma(\chi):=\chi^{p}-(\chi-1)^{p} .
\end{gathered}
$$

In this paper, we consider the explicit 2-step mixed method for the RLW equation. For $p=2$, we obtained easily

$$
\begin{gathered}
\alpha_{0}=\frac{3}{2}, \quad \alpha_{1}=-2, \quad \alpha_{2}=\frac{1}{2}, \\
\sigma_{0}=-1, \quad \sigma_{1}=2 .
\end{gathered}
$$

Substituting (19) into (17), we obtain the following 2-step mixed scheme:

$$
\begin{gathered}
(A+\beta B)\left(\frac{3}{2} Z^{n+2}-2 Z^{n+1}+\frac{1}{2} Z^{n}\right) \\
=\Delta t\left[\gamma C\left(2 Z^{n+1}-Z^{n}\right)+\delta\left(2 D\left(U^{n+1}\right) Z^{n+1}\right.\right. \\
\left.\left.-D\left(U^{n}\right) Z^{n}\right)\right], \\
B U^{n+2}=C Z^{n+2} .
\end{gathered}
$$

Remark 1. There have been many numerical schemes for the RLW equation, but we have not seen the related research on explicit multistep mixed element method for RLW equation in the literature. From the viewpoint of numerical theory, we propose a mixed element scheme (6) and (7), which is different from some other mixed finite element methods in [14-16], for the RLW equation and derive some a priori error estimates based on the explicit multistep mixed element method. From the perspective of numerical calculation, our method is efficient for RLW equation.

\section{Two-Step Mixed Scheme and Optimal Error Estimates}

3.1. Two-Step Mixed Scheme and Some Lemmas. In this section, we will discuss some a priori error estimates based on explicit 2-step mixed finite element method for the RLW equation. For the fully discrete procedure, let $0=t_{0}<t_{1}<$ $\cdots<t_{N}=T$ be a given partition of the time interval $[0, T]$ with step length $\Delta t=T / N$, for some positive integer $N$. For a smooth function $\phi$ on $[0, T]$, define $\phi^{n}=\phi\left(t_{n}\right)$.

The system (3) and (4) has the following formulation at $t=t_{n+1}$ :

$$
\begin{gathered}
\left(u_{x}^{n+1}, v_{x}\right)=\left(q^{n+1}, v_{x}\right), \quad \forall v \in H_{0}^{1} \\
\left(q_{t}^{n+1}, w\right)+\beta\left(q_{x t}^{n+1}, w_{x}\right)=\gamma\left(q^{n+1}, w_{x}\right)+\delta\left(u^{n+1} q^{n+1}, w_{x}\right),
\end{gathered}
$$

$\forall w \in H^{1}$.

Based on system (17), we get an equivalent formulation for system (21) as

$$
\left(u_{x}^{n+1}, v_{x}\right)=\left(q^{n+1}, v_{x}\right), \quad \forall v \in H_{0}^{1},
$$

$$
\begin{gathered}
\left(\frac{3 q^{n+1}-4 q^{n}+q^{n-1}}{2 \Delta t}, w\right)+\beta\left(\frac{3 q_{x}^{n+1}-4 q_{x}^{n}+q_{x}^{n-1}}{2 \Delta t}, w_{x}\right) \\
=-\left(\tau^{n+1}, w\right)-\beta\left(\kappa_{1}^{n+1}, w_{x}\right)+\gamma\left(2 q^{n}-q^{n-1}, w_{x}\right) \\
\quad+\delta\left(2 u^{n} q^{n}-u^{n-1} q^{n-1}, w_{x}\right)+\gamma\left(R_{1}^{n+1}, w_{x}\right) \\
+\delta\left(R_{2}^{n+1}, w_{x}\right), \quad \forall w \in H^{1}
\end{gathered}
$$


TABLE 5: Convergence order and error in $L^{\infty}$ norm for $q$ of time with $h=0.125$ and $c=0.1$.

\begin{tabular}{lccccc}
\hline Time & $\Delta t=0.4$ & $\Delta t=0.2$ & $\Delta t=0.1$ & Order $(0.2 / 0.4)$ & Order $(0.1 / 0.2)$ \\
\hline 4 & $1.1595 e-004$ & $2.7265 e-005$ & $6.3127 e-006$ & 2.0884 & 2.1107 \\
8 & $1.9712 e-004$ & $4.6625 e-005$ & $1.0725 e-005$ & 2.0799 & 2.1201 \\
12 & $2.5295 e-004$ & $5.9837 e-005$ & $1.3579 e-005$ & 2.0797 & 2.1397 \\
16 & $2.9012 e-004$ & $6.8641 e-005$ & $1.5594 e-005$ & 2.0795 & 2.1381 \\
20 & $3.1768 e-004$ & $7.5864 e-005$ & $1.7195 e-005$ & 2.0661 & 2.1414 \\
\hline
\end{tabular}

TABLE 6: Convergence order and error in $L^{2}$ norm for $u$ of space with $\Delta t=0.01$ and $c=0.1$.

\begin{tabular}{lccccc}
\hline Time & $h=0.8$ & $h=0.4$ & $h=0.2$ & Order $(0.4 / 0.8)$ & Order $(0.2 / 0.4)$ \\
\hline 4 & $1.7109 e-003$ & $4.2677 e-004$ & $1.1449 e-004$ & 1.9940 & 1.8982 \\
8 & $1.8945 e-003$ & $4.6862 e-004$ & $1.1924 e-004$ & 2.0195 & 1.9746 \\
12 & $2.1232 e-003$ & $5.2130 e-004$ & $1.3025 e-004$ & 2.0102 & 2.0008 \\
16 & $2.3559 e-003$ & $5.7598 e-004$ & $1.4421 e-004$ & 2.0589 & 1.9978 \\
20 & $2.5778 e-003$ & $6.3407 e-004$ & $1.7877 e-004$ & 2.0358 & 1.8265 \\
\hline
\end{tabular}

where

$$
\begin{gathered}
\tau^{n+1}=q_{t}^{n+1}-\frac{3 q^{n+1}-4 q^{n}+q^{n-1}}{2 \Delta t}, \\
\kappa_{1}^{n+1}=q_{x t}\left(t_{n+1}\right)-\frac{3 q_{x}^{n+1}-4 q_{x}^{n}+q_{x}^{n-1}}{2 \Delta t}, \\
R_{1}^{n+1}=q^{n+1}-\left(2 q^{n}-q^{n-1}\right), \\
R_{2}^{n+1}=u^{n+1} q^{n+1}-\left(2 u^{n} q^{n}-u^{n-1} q^{n-1}\right) .
\end{gathered}
$$

We now find a pair $\left\{U^{n+1}, Z^{n+1}\right\}$ in $V_{h} \times W_{h}$ satisfying

$$
\begin{gathered}
\left(U_{x}^{n+1}, v_{x}^{h}\right)=\left(Z^{n+1}, v_{x}^{h}\right), \quad \forall v^{h} \in V_{h}, \\
\left(\frac{3 Z^{n+1}-4 Z^{n}+Z^{n-1}}{2 \Delta t}, w^{h}\right)+\beta\left(\frac{3 Z_{x}^{n+1}-4 Z_{x}^{n}+Z_{x}^{n-1}}{2 \Delta t}, w_{x}^{h}\right) \\
=\gamma\left(2 Z^{n}-Z^{n-1}, w_{x}^{h}\right)+\delta\left(2 U^{n} Z^{n}-U^{n-1} Z^{n-1}, w_{x}^{h}\right), \\
\forall w^{h} \in W_{h} .
\end{gathered}
$$

For the theoretical analysis of a priori error estimates, we define the following projections.

Lemma 2 (see $[15,27,28])$. One defines the elliptic projection $\tilde{u}^{h} \in V_{h}$ by

$$
\left(u_{x}-\widetilde{u}_{x}^{h}, v_{x}^{h}\right)=0, \quad v^{h} \in V_{h}
$$

With $\eta=u-\tilde{u}^{h}$, the following estimates are well known for $j=0,1$ :

$$
\|\eta\|_{j} \leq C h^{k+1-j}\|u\|_{k+1}
$$

Lemma 3 (see $[15,27,28]$ ). Furthermore, one also defines a Ritz projection $\widetilde{q}^{h} \in W_{h}$ of $q$ as the solution of

$$
A\left(q-\widehat{q}^{h}, w^{h}\right)=0, \quad w^{h} \in W_{h},
$$

where $A(q, w)=\left(q_{x}, w_{x}\right)+\lambda(q, w)$, and $\lambda$ is taken appropriately so that

$$
A(w, w) \geq \mu_{0}\|w\|_{1}^{2}, \quad w \in H^{1},
$$

where $\mu_{0}$ is a positive constant. Moreover, it is easy to verify that $A(\cdot, \cdot)$ is bounded.

With $\rho=q-\widetilde{q}^{h}$, the following estimates hold:

$$
\left\|\frac{\partial^{i} \rho}{\partial t^{i}}\right\|_{j} \leq C h^{r+1-j}\left\|\frac{\partial^{i} q}{\partial t^{i}}\right\|_{r+1}, \quad i=0,1,2,3 ; j=0,1 .
$$

For fully discrete error estimates, we now write the errors as

$$
\begin{gathered}
u\left(t_{n}\right)-U^{n}=\left(u\left(t_{n}\right)-\tilde{u}^{h}\left(t_{n}\right)\right)+\left(\tilde{u}^{h}\left(t_{n}\right)-U^{n}\right)=\eta^{n}+\varsigma^{n}, \\
q\left(t_{n}\right)-Z^{n}=\left(q\left(t_{n}\right)-\widetilde{q}^{h}\left(t_{n}\right)\right) \\
+\left(\widetilde{q}^{h}\left(t_{n}\right)-Z^{n}\right)=\rho^{n}+\xi^{n} .
\end{gathered}
$$

Combine (27), (29), (22), (23), (25), and (26) at $t=t_{n+1}$ to get the following error equations:

$$
\begin{gathered}
\left(\varsigma_{x}^{n+1}, v_{x}^{h}\right)=\left(\rho^{n+1}+\xi^{n+1}, v_{x}^{h}\right), \quad \forall v^{h} \in V_{h}, \\
\left(\frac{3 \xi^{n+1}-4 \xi^{n}+\xi^{n-1}}{2 \Delta t}, w^{h}\right)+\beta\left(\frac{3 \xi_{x}^{n+1}-4 \xi_{x}^{n}+\xi_{x}^{n-1}}{2 \Delta t}, w_{x}^{h}\right) \\
=-\left((1-\beta \lambda) \frac{3 \rho^{n+1}-4 \rho^{n}+\rho^{n-1}}{2 \Delta t}+\tau^{n+1}, w^{h}\right) \\
-\beta\left(\kappa_{2}^{n+1}, w_{x}^{h}\right)+\gamma\left(2 \xi^{n}-\xi^{n-1}, w_{x}^{h}\right) \\
+\delta\left(2\left(u\left(t_{n}\right) q\left(t_{n}\right)-U^{n} Z^{n}\right)\right. \\
\left.\quad-\left(u\left(t_{n-1}\right) q\left(t_{n-1}\right)-U^{n-1} Z^{n-1}\right), w_{x}^{h}\right) \\
+\gamma\left(R_{1}^{n+1}, w_{x}^{h}\right)+\delta\left(R_{2}^{n+1}, w_{x}^{h}\right), \quad \forall w^{h} \in W_{h}
\end{gathered}
$$


TABLE 7: Convergence order and error in $L^{\infty}$ norm for $u$ of space with $\Delta t=0.01$ and $c=0.1$.

\begin{tabular}{lccccc}
\hline Time & $h=0.8$ & $h=0.4$ & $h=0.2$ & Order $(0.4 / 0.8)$ & Order $(0.2 / 0.4)$ \\
\hline 4 & $2.1763 e-003$ & $5.8447 e-004$ & $1.5502 e-004$ & 1.8967 & 1.9147 \\
8 & $4.7892 e-003$ & $1.2098 e-003$ & $3.0533 e-004$ & 1.9850 & 1.9863 \\
12 & $7.2371 e-003$ & $1.7871 e-003$ & $4.4434 e-004$ & 2.0178 & 2.0079 \\
16 & $9.4746 e-003$ & $2.3084 e-003$ & $5.7081 e-004$ & 2.0372 & 2.0158 \\
20 & $1.1534 e-002$ & $2.7859 e-003$ & $6.8980 e-004$ & 2.0497 & 2.0139 \\
\hline
\end{tabular}

TABLE 8: Convergence order and error in $L^{2}$ norm for $q$ of space with $\Delta t=0.01$ and $c=0.1$.

\begin{tabular}{lccccc}
\hline Time & $h=0.8$ & $h=0.4$ & $h=0.2$ & Order $(0.4 / 0.8)$ & Order $(0.2 / 0.4)$ \\
\hline 4 & $2.3426 e-004$ & $5.4759 e-005$ & $1.2581 e-005$ & 2.0969 & 2.1218 \\
8 & $4.2831 e-004$ & $1.0038 e-004$ & $2.2853 e-005$ & 2.0932 & 2.1350 \\
12 & $5.7270 e-004$ & $1.3452 e-004$ & $3.0443 e-005$ & 2.0900 & 2.1436 \\
16 & $6.7812 e-004$ & $1.5951 e-004$ & $3.5927 e-005$ & 2.0879 & 2.1505 \\
20 & $7.5693 e-004$ & $1.7832 e-004$ & $4.0176 e-005$ & 2.0857 & 2.1501 \\
\hline
\end{tabular}

where

$$
\kappa_{2}^{n+1}=\widetilde{q}_{x t}^{h}\left(t_{n+1}\right)-\frac{3 \widetilde{q}_{x}^{h, n+1}-4 \widetilde{q}_{x}^{h, n}+\widetilde{q}_{x}^{h, n-1}}{2 \Delta t} .
$$

Lemma 4. For $\tau^{n+1}, \kappa_{2}^{n+1}, R_{1}^{n+1}$, and $R_{2}^{n+1}$, the following estimates hold:

$$
\begin{gathered}
\left\|\tau^{n+1}\right\| \leq C \Delta t^{2}\left\|q_{t t t}\right\|_{L^{\infty}\left(L^{2}\right)}, \\
\left\|\kappa_{2}^{n+1}\right\| \leq C \Delta t^{2}\left(h^{r}\left\|q_{x t t t}\right\|_{L^{\infty}\left(H^{r+1}\right)}+\left\|q_{x t t t}\right\|_{L^{\infty}\left(L^{2}\right)}\right), \\
\left\|R_{1}^{n+1}\right\| \leq C \Delta t^{2}\left\|q_{t t}\right\|_{L^{\infty}\left(L^{2}\right)}, \\
\left\|R_{2}^{n+1}\right\| \leq C \Delta t^{2}\left(\left\|u q_{t t}\right\|_{L^{\infty}\left(L^{2}\right)}+\left\|u_{t} q_{t}\right\|_{L^{\infty}\left(L^{2}\right)}+\left\|u_{t t} q\right\|_{L^{\infty}\left(L^{2}\right)}\right) .
\end{gathered}
$$

Proof. Using the Taylor expansion, we have

$$
\begin{aligned}
q\left(t_{n}\right)= & q\left(t_{n+1}\right)+q_{t}\left(t_{n+1}\right)\left(t_{n}-t_{n+1}\right) \\
& +\frac{q_{t t}\left(t_{\Delta 1}\right)}{2}\left(t_{n}-t_{n+1}\right)^{2}, \quad t_{n}<t_{\Delta 1}<t_{n+1}, \\
q\left(t_{n-1}\right)= & q\left(t_{n+1}\right)+q_{t}\left(t_{n+1}\right)\left(t_{n-1}-t_{n+1}\right) \\
& +\frac{q_{t t}\left(t_{\Delta 2}\right)}{2}\left(t_{n-1}-t_{n+1}\right)^{2}, \quad t_{n-1}<t_{\Delta 2}<t_{n+1} .
\end{aligned}
$$

Combining (37) and (38) and noting that $-2 \Delta t=2\left(t_{n}-\right.$ $\left.t_{n+1}\right)=t_{n-1}-t_{n+1}$, we obtain

$$
q\left(t_{n+1}\right)=2 q\left(t_{n}\right)-q\left(t_{n-1}\right)+\left(q_{t t}\left(t_{\Delta 1}\right)-2 q_{t t}\left(t_{\Delta 2}\right)\right) \Delta t^{2} .
$$

From (39), we have

$$
\left\|q\left(t_{n+1}\right)-\left(2 q\left(t_{n}\right)-q\left(t_{n-1}\right)\right)\right\| \leq C \Delta t^{2}\left\|q_{t t}\right\|_{L^{\infty}\left(L^{2}\right)} .
$$

Using a similar estimate as the one for $\left\|R_{1}^{n+1}\right\|$, we have

$$
\begin{aligned}
u\left(t_{n+1}\right) q\left(t_{n+1}\right)= & 2 u\left(t_{n}\right) q\left(t_{n}\right)-u\left(t_{n-1}\right) q\left(t_{n-1}\right) \\
& +\left((u q)_{t t}\left(t_{\Delta 3}\right)-2(u q)_{t t}\left(t_{\Delta 4}\right)\right) \Delta t^{2}
\end{aligned}
$$

where $t_{n}<t_{\Delta 3}<t_{n+1}, t_{n-1}<t_{\Delta 4}<t_{n+1}$.

From (41), we have

$$
\begin{aligned}
& \left\|u\left(t_{n+1}\right) q\left(t_{n+1}\right)-\left(2 u\left(t_{n}\right) q\left(t_{n}\right)-u\left(t_{n-1}\right) q\left(t_{n-1}\right)\right)\right\| \\
& \quad \leq C \Delta t^{2}\left(\left\|u q_{t t}\right\|_{L^{\infty}\left(L^{2}\right)}+\left\|u_{t} q_{t}\right\|_{L^{\infty}\left(L^{2}\right)}+\left\|u_{t t} q\right\|_{L^{\infty}\left(L^{2}\right)}\right) .
\end{aligned}
$$

Using the Taylor expansion and noting that $-2 \Delta t=2\left(t_{n}-\right.$ $\left.t_{n+1}\right)=t_{n-1}-t_{n+1}$, we have

$$
\begin{aligned}
4 q\left(t_{n}\right)= & 4 q\left(t_{n+1}\right)-4 q_{t}\left(t_{n+1}\right) \Delta t+2 q_{t t}\left(t_{n+1}\right) \Delta t^{2} \\
& -\frac{2 q_{t t t}\left(t_{\Delta 5}\right)}{3} \Delta t^{3}, \quad t_{n}<t_{\Delta 5}<t_{n+1}, \\
q\left(t_{n-1}\right)= & q\left(t_{n+1}\right)-2 q_{t}\left(t_{n+1}\right) \Delta t+2 \frac{q_{t t}\left(t_{n+1}\right)}{2} \Delta t^{2} \\
& -\frac{4 q_{t t t}\left(t_{\Delta 6}\right)}{3} \Delta t^{3}, \quad t_{n-1}<t_{\Delta 6}<t_{n+1} .
\end{aligned}
$$

Using (43), we obtain

$$
q_{t}^{n+1}=\frac{3 q^{n+1}-4 q^{n}+q^{n-1}}{2 \Delta t}-\left(\frac{2 q_{t t t}\left(t_{\Delta 5}\right)}{3}-\frac{4 q_{t t t}\left(t_{\Delta 6}\right)}{3}\right) \Delta t^{2}
$$

By (44), we have

$$
\left\|\tau^{n+1}\right\| \leq C \Delta t^{2}\left\|q_{t t t}(t)\right\|_{L^{\infty}\left(L^{2}\right)}
$$


TABLE 9: Convergence order and error in $L^{\infty}$ norm for $q$ of space with $\Delta t=0.01$ and $c=0.1$.

\begin{tabular}{lccccc}
\hline Time & $h=0.8$ & $h=0.4$ & $h=0.2$ & Order $(0.4 / 0.8)$ & Order $(0.2 / 0.4)$ \\
\hline 4 & $1.1957 e-003$ & $3.1367 e-004$ & $7.8812 e-005$ & 1.9305 & 1.9928 \\
8 & $2.4344 e-003$ & $6.0206 e-004$ & $1.4825 e-004$ & 2.0156 & 2.0219 \\
12 & $3.4768 e-003$ & $8.3962 e-004$ & $2.0540 e-004$ & 2.0500 & 2.0313 \\
16 & $4.3743 e-003$ & $1.0402 e-003$ & $2.5371 e-004$ & 2.0722 & 2.0356 \\
20 & $5.1677 e-003$ & $1.2153 e-003$ & $2.9544 e-004$ & 2.0882 & 2.0404 \\
\hline
\end{tabular}

Using the similar method to the estimate for $\left\|\tau^{n+1}\right\|$ and (31), we obtain

$$
\begin{aligned}
\left\|\kappa_{2}^{n+1}\right\| & \leq C \Delta t^{2}\left\|\vec{q}_{x t t t}^{h}\right\|_{L^{\infty}\left(L^{2}\right)} \\
& \leq C \Delta t^{2}\left(\left\|\rho_{x t t}\right\|_{L^{\infty}\left(L^{2}\right)}+\left\|q_{x t t t}\right\|_{L^{\infty}\left(L^{2}\right)}\right) \\
& \leq C \Delta t^{2}\left(h^{r}\left\|q_{x t t t}\right\|_{L^{\infty}\left(H^{r+1}\right)}+\left\|q_{x t t t}\right\|_{L^{\infty}\left(L^{2}\right)}\right) .
\end{aligned}
$$

3.2. Optimal Error Estimates. In this subsection, we derive the fully discrete optimal error estimates and obtain the following theorem.

Theorem 5. Assuming that $U^{0}, U^{1} \in V_{h}$ and $Z^{0}, Z^{1} \in W_{h}$ are given, then for $1 \leq J \leq M, j=0,1$, one has

$$
\begin{gathered}
\left\|u^{J+1}-U^{J+1}\right\|_{j} \leq C\left(h^{\min (k+1-j, r+1)}+\Delta t^{2}\right), \\
\left\|q^{J+1}-Z^{J+1}\right\|_{j}+\left\|2\left(q^{J+1}-Z^{J+1}\right)-\left(q^{J}-Z^{J}\right)\right\|_{j} \\
\leq C\left(h^{\min (k+1, r+1-j)}+\Delta t^{2}\right) .
\end{gathered}
$$

Proof. Taking $v^{h}=\varsigma^{n+1}$ in (33) and using Cauchy-Schwarz's inequality and Poincaré's inequality, we get

$$
\left\|\varsigma^{n+1}\right\| \leq C\left\|\varsigma_{x}^{n+1}\right\| \leq C\left(\left\|\rho^{n+1}\right\|+\left\|\xi^{n+1}\right\|\right)
$$

Set $w^{h}=\xi^{n+1}$ in (34) to obtain

$$
\begin{aligned}
\left(\frac{3 \xi^{n+1}-4 \xi^{n}+\xi^{n-1}}{2 \Delta t}, \xi^{n+1}\right)+\beta\left(\frac{3 \xi_{x}^{n+1}-4 \xi_{x}^{n}+\xi_{x}^{n-1}}{2 \Delta t}, \xi_{x}^{n+1}\right) \\
=-\left((1-\beta \lambda) \frac{3 \rho^{n+1}-4 \rho^{n}+\rho^{n-1}}{2 \Delta t}+\tau^{n+1}, \xi^{n+1}\right) \\
-\beta\left(\kappa^{n+1}, \xi_{x}^{n+1}\right)+\gamma\left(2 \rho^{n}-\rho^{n-1}, \xi_{x}^{n+1}\right) \\
+\gamma\left(2 \xi^{n}-\xi^{n-1}, \xi_{x}^{n+1}\right)+\gamma\left(R_{1}^{n+1}, \xi_{x}^{n+1}\right)+\delta\left(R_{2}^{n+1}, \xi_{x}^{n+1}\right) \\
+\delta\left(2\left(u\left(t_{n}\right) q\left(t_{n}\right)-U^{n} Z^{n}\right)\right. \\
\left.\quad-\left(u\left(t_{n-1}\right) q\left(t_{n-1}\right)-U^{n-1} Z^{n-1}\right), \xi_{x}^{n+1}\right) .
\end{aligned}
$$

Use (48) as well as the Cauchy-Schwarz and Young's inequalities to obtain

$$
\begin{gathered}
\left(\frac{3 \xi^{n+1}-4 \xi^{n}+\xi^{n-1}}{2 \Delta t}, \xi^{n+1}\right)+\beta\left(\frac{3 \xi_{x}^{n+1}-4 \xi_{x}^{n}+\xi_{x}^{n-1}}{2 \Delta t}, \xi_{x}^{n+1}\right) \\
\leq C\left(\left\|\xi^{n+1}\right\|^{2}+\left\|\xi_{x}^{n+1}\right\|^{2}+\left\|\xi^{n}\right\|^{2}+\left\|2 \rho^{n}-\rho^{n-1}\right\|^{2}\right. \\
+\left\|2 \xi^{n}-\xi^{n-1}\right\|^{2}+\left\|\frac{3 \rho^{n+1}-4 \rho^{n}+\rho^{n-1}}{2 \Delta t}\right\|^{2} \\
\left.+\left\|\tau^{n+1}\right\|^{2}+\left\|\kappa^{n+1}\right\|^{2}+\left\|R_{1}^{n+1}\right\|^{2}+\left\|R_{2}^{n+1}\right\|^{2}\right) \\
+\delta \mid\left(2\left(u\left(t_{n}\right) q\left(t_{n}\right)-U^{n} Z^{n}\right)\right. \\
\left.-\left(u\left(t_{n-1}\right) q\left(t_{n-1}\right)-U^{n-1} Z^{n-1}\right), \xi_{x}^{n+1}\right) \mid .
\end{gathered}
$$

Note that

$$
\text { (a) } \begin{array}{r}
\left(\frac{3 \xi^{n+1}-4 \xi^{n}+\xi^{n-1}}{2 \Delta t}, \xi^{n+1}\right) \\
=\frac{1}{4 \Delta t}\left[\left(\left\|\xi^{n+1}\right\|^{2}+\left\|2 \xi^{n+1}-\xi^{n}\right\|^{2}\right)\right. \\
-\left(\left\|\xi^{n}\right\|^{2}+\left\|2 \xi^{n}-\xi^{n-1}\right\|^{2}\right) \\
\left.+\left\|\xi^{n+1}-2 \xi^{n}+\xi^{n-1}\right\|^{2}\right]
\end{array}
$$

(b) $\beta\left(\frac{3 \xi_{x}^{n+1}-4 \xi_{x}^{n}+\xi_{x}^{n-1}}{2 \Delta t}, \xi_{x}^{n+1}\right)$

$$
=\frac{1}{4 \Delta t}\left[\left(\left\|\xi_{x}^{n+1}\right\|^{2}+\left\|2 \xi_{x}^{n+1}-\xi_{x}^{n}\right\|^{2}\right)\right.
$$$$
-\left(\left\|\xi_{x}^{n}\right\|^{2}+\left\|2 \xi_{x}^{n}-\xi_{x}^{n-1}\right\|^{2}\right)
$$$$
\left.+\left\|\xi_{x}^{n+1}-2 \xi_{x}^{n}+\xi_{x}^{n-1}\right\|^{2}\right]
$$

(c) $\left\|\frac{3 \rho^{n+1}-4 \rho^{n}+\rho^{n-1}}{2 \Delta t}\right\|^{2} \leq \frac{3}{2 \Delta t} \int_{t_{n}}^{t_{n+1}}\left\|\rho_{t}\right\|^{2} d s$

$$
+\frac{1}{2 \Delta t} \int_{t_{n-1}}^{t_{n}}\left\|\rho_{t}\right\|^{2} d s
$$




$$
\begin{aligned}
\mid(2 & \left(u\left(t_{n}\right) q\left(t_{n}\right)-U^{n} Z^{n}\right) \\
- & \left.\left(u\left(t_{n-1}\right) q\left(t_{n-1}\right)-U^{n-1} Z^{n-1}\right), \xi_{x}^{n+1}\right) \mid \\
\leq & \left|\left(2 u\left(t_{n}\right)\left(q\left(t_{n}\right)-Z^{n}\right)-u\left(t_{n}\right)\left(q\left(t_{n-1}\right)-Z^{n-1}\right), \xi_{x}^{n+1}\right)\right| \\
& +\left|\left(\left(u\left(t_{n}\right)-u\left(t_{n-1}\right)\right)\left(q\left(t_{n-1}\right)-Z^{n-1}\right), \xi_{x}^{n+1}\right)\right| \\
& +\left|\left(2\left(u\left(t_{n}\right)-U^{n}\right) Z^{n}-\left(u\left(t_{n-1}\right)-U^{n-1}\right) Z^{n}, \xi_{x}^{n+1}\right)\right| \\
& +\left|\left(\left(u\left(t_{n-1}\right)-U^{n-1}\right)\left(Z^{n}-Z^{n-1}\right), \xi_{x}^{n+1}\right)\right| \\
\doteq & T_{1}+T_{2}+T_{3}+T_{4} .
\end{aligned}
$$

We now estimate $T_{1}, T_{2}, T_{3}$, and $T_{4}$ as

$$
\begin{aligned}
& T_{1} \leq\left|\left(u\left(t_{n}\right)\left(2 \xi^{n}-\xi^{n-1}\right), \xi_{x}^{n+1}\right)\right| \\
&+\left|\left(u\left(t_{n}\right)\left(2 \rho^{n}-\rho^{n-1}\right), \xi_{x}^{n+1}\right)\right| \\
& \leq\left\|u\left(t_{n}\right)\right\|_{\infty}\left\|2 \xi^{n}-\xi^{n-1}\right\|\left\|\xi_{x}^{n+1}\right\| \\
&+\left\|u\left(t_{n}\right)\right\|_{\infty}\left\|2 \rho^{n}-\rho^{n-1}\right\|\left\|\xi_{x}^{n+1}\right\|, \\
& T_{2}=\left|\left(\int_{t_{n-1}}^{t_{n}} u_{t} d s\left(\rho^{n-1}+\xi^{n-1}\right), \xi_{x}^{n+1}\right)\right| \\
& \leq \Delta t\left\|u_{t}\right\|_{\infty}\left\|\rho^{n-1}+\xi^{n-1}\right\|\left\|\xi_{x}^{n+1}\right\|, \\
& T_{3} \leq\left|\left(Z^{n}\left(2 \varsigma^{n}-\varsigma^{n-1}\right), \xi_{x}^{n+1}\right)\right| \\
&+\left|\left(Z^{n}\left(2 \eta^{n}-\eta^{n-1}\right), \xi_{x}^{n+1}\right)\right| \\
& \leq\left\|Z^{n}\right\|_{\infty}\left\|2 \varsigma^{n}-\varsigma^{n-1}\right\|\left\|\xi_{x}^{n+1}\right\| \\
&+\left\|Z^{n}\right\|_{\infty}\left\|2 \eta^{n}-\eta^{n-1}\right\|\left\|\xi_{x}^{n+1}\right\|, \\
& T_{4} \leq\left|\left(\int_{t_{n-1}}^{t_{n}} Z_{t} d s\left(\eta^{n-1}+\varsigma^{n-1}\right), \xi_{x}^{n+1}\right)\right| \\
& \leq \Delta t\left\|Z_{t}\right\|\left\|_{\infty}\right\| \eta^{n-1}+\varsigma^{n-1}\|\| \xi_{x}^{n+1} \| .
\end{aligned}
$$

Substituting (53) into (52), we obtain

$$
\begin{gathered}
\mid\left(2\left(u\left(t_{n}\right) q\left(t_{n}\right)-U^{n} Z^{n}\right)\right. \\
\left.-\left(u\left(t_{n-1}\right) q\left(t_{n-1}\right)-U^{n-1} Z^{n-1}\right), \xi_{x}^{n+1}\right) \mid \\
\leq C\left(\left\|2 \xi^{n}-\xi^{n-1}\right\|^{2}+\left\|\xi^{n-1}\right\|^{2}\right. \\
+\left\|\rho^{n}\right\|^{2}+\left\|\rho^{n-1}\right\|^{2}+\left\|\varsigma^{n}\right\|^{2}+\left\|\varsigma^{n-1}\right\|^{2} \\
\left.+\left\|\eta^{n}\right\|^{2}+\left\|\eta^{n-1}\right\|^{2}+\left\|\xi_{x}^{n+1}\right\|^{2}\right) .
\end{gathered}
$$

Substituting (36), (51), and (54) into (50), using (48), and summing from $n=1,2, \ldots, J$, the resulting inequality becomes

$$
\begin{gathered}
(1-C \Delta t)\left(\left\|\xi^{J+1}\right\|^{2}+\left\|2 \xi^{J+1}-\xi^{J}\right\|^{2}+\left\|\xi_{x}^{J+1}\right\|^{2}\right. \\
\left.+\left\|2 \xi_{x}^{J+1}-\xi_{x}^{J}\right\|^{2}\right) \\
\leq C \Delta t \sum_{n=1}^{J}\left(\left\|\eta^{n-1}\right\|^{2}+\left\|\rho^{n-1}\right\|^{2}\right)+C \int_{0}^{t_{J+1}}\left\|\rho_{t}\right\|^{2} d s \\
+C \Delta t^{4}\left(\left\|q_{t t}\right\|_{L^{\infty}\left(L^{2}\right)}^{2}+\left\|q_{t t t}\right\|_{L^{\infty}\left(L^{2}\right)}^{2}\right. \\
+\left\|u q_{t t}\right\|_{L^{\infty}\left(L^{2}\right)}+\left\|u_{t} q_{t}\right\|_{L^{\infty}\left(L^{2}\right)}+\left\|u_{t t} q\right\|_{L^{\infty}\left(L^{2}\right)} \\
\left.+h^{r}\left\|q_{x t t t}\right\|_{L^{\infty}\left(H^{+1}\right)}+\left\|q_{x t t t}\right\|_{L^{\infty}\left(L^{2}\right)}\right) \\
+C \Delta t \sum_{n=1}^{J}\left(\left\|\xi^{n}\right\|^{2}+\left\|\xi_{x}^{n}\right\|^{2}+\left\|2 \xi^{n}-\xi^{n-1}\right\|^{2}\right. \\
\left.+\left\|2 \xi_{x}^{n}-\xi_{x}^{n-1}\right\|^{2}\right) .
\end{gathered}
$$

Choose $\Delta t_{0}$ in such a way that for $0<\Delta t \leq \Delta t_{0},(1-C \Delta t)>0$. Then, as an application of Gronwall's lemma, we obtain

$$
\begin{aligned}
\left\|\xi^{J+1}\right\|^{2}+\left\|2 \xi^{J+1}-\xi^{J}\right\|^{2}+\left\|\xi_{x}^{J+1}\right\|^{2}+\left\|2 \xi_{x}^{J+1}-\xi_{x}^{J}\right\|^{2} \\
\leq C \Delta t \sum_{n=1}^{J}\left(\left\|\eta^{n-1}\right\|^{2}+\left\|\rho^{n-1}\right\|^{2}\right)+C \int_{0}^{t_{J+1}}\left\|\rho_{t}\right\|^{2} d s \\
+C \Delta t^{4}\left(\left\|q_{t t}\right\|_{L^{\infty}\left(L^{2}\right)}^{2}+\left\|q_{t t t}\right\|_{L^{\infty}\left(L^{2}\right)}^{2}+\left\|u q_{t t}\right\|_{L^{\infty}\left(L^{2}\right)}\right. \\
+\left\|u_{t} q_{t}\right\|_{L^{\infty}\left(L^{2}\right)}+\left\|u_{t t} q\right\|_{L^{\infty}\left(L^{2}\right)} \\
\left.+h^{r}\left\|q_{x t t t}\right\|_{L^{\infty}\left(H^{r+1}\right)}+\left\|q_{x t t t}\right\|_{L^{\infty}\left(L^{2}\right)}\right)
\end{aligned}
$$

Combine (28), (31), and (56) with the triangle inequality to complete the $L^{2}$ and $H^{1}$ error estimates for $q$. Furthermore, use (48) and the triangle inequality to complete the optimal error estimates for $\left\|u\left(t_{n}\right)-U^{n}\right\|$ and $\left\|u\left(t_{n}\right)-U^{n}\right\|_{1}$.

Remark 6. Compared to a variety of difference methods in [17], our method is studied based on mixed element scheme (6) and (7).

Remark 7. Although some convergence proofs of multistep methods for RLW/BBM are provided in [20, 25, 29], our convergence results of multistep methods are proved based on a mixed finite element scheme. Based on the current discussion, we have to provide the detailed proofs for multistep mixed finite element methods in this paper. 


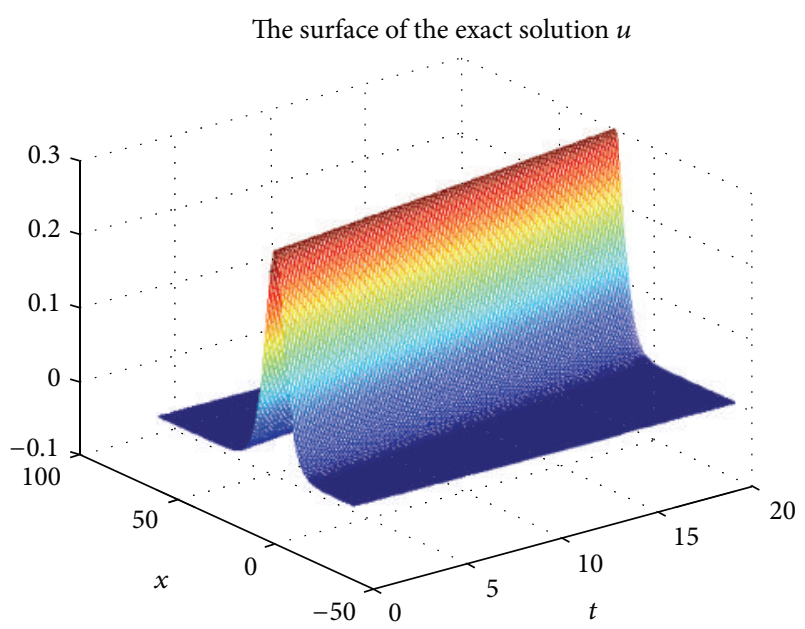

FIGURE 1: Surface for exact solution $u$.

The surface of the numerical solution $u_{h}$

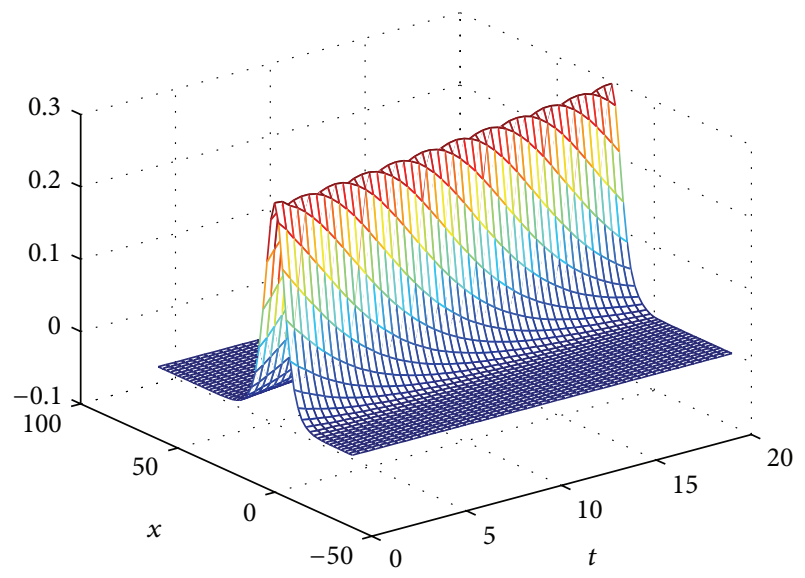

FIGURE 2: Surface for numerical solution $u_{h}$.

\section{Numerical Results}

In order to test the viability of the proposed method, we consider a test problem. We write conservation laws as $[3,4]$

$$
\begin{gathered}
Q_{1}=\int_{a}^{b} u d x \simeq h \sum_{j=1}^{N} u_{j}^{n}, \\
Q_{2}=\int_{a}^{b}\left(u^{2}+\mu\left(u_{x}\right)^{2}\right) d x \simeq h \sum_{j=1}^{N}\left[\left(u_{j}^{n}\right)^{2}+\mu\left[\left(u_{x}\right)_{j}^{n}\right]^{2}\right], \\
Q_{3}=\int_{a}^{b}\left(u^{3}+3 u^{2}\right) d x \simeq h \sum_{j=1}^{N}\left[\left(u_{j}^{n}\right)^{3}+3\left[(u)_{j}^{n}\right]^{2}\right],
\end{gathered}
$$

where $Q_{1}, Q_{2}$, and $Q_{3}$ are usually called mass, momentum, and energy, respectively, which are observed to check the conservation of the numerical scheme.

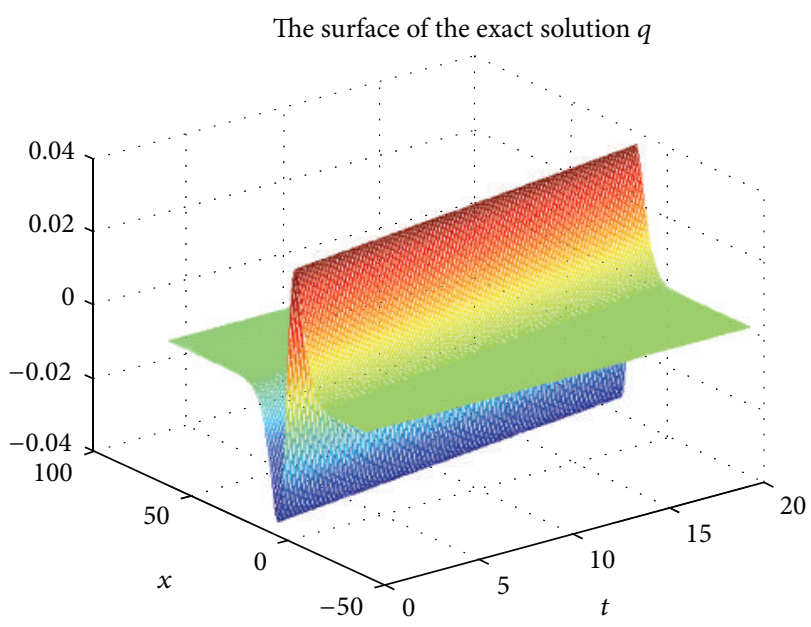

FIGURE 3: Surface for exact solution $q$.

The surface of the numerical solution $q_{h}$

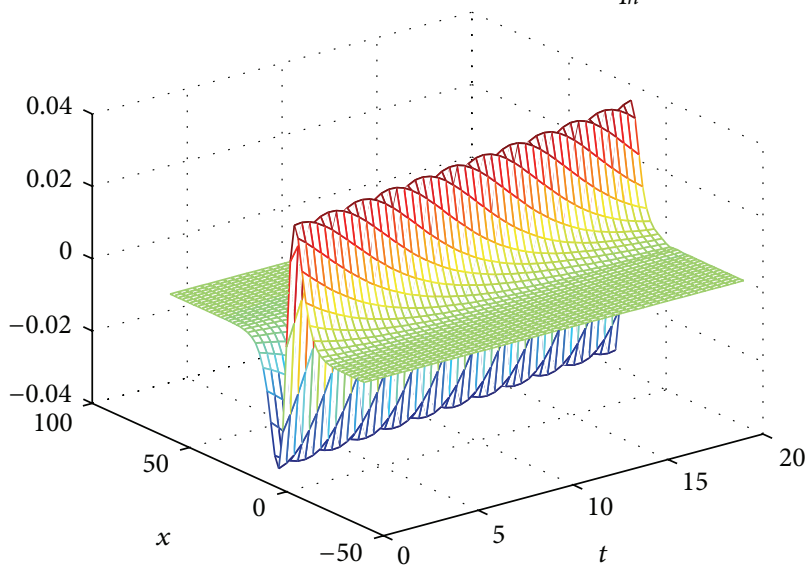

FIGURE 4: Surface for numerical solution $q_{h}$.

We consider RLW equation (1) and let, in (1), $\delta=\gamma=\beta=$ 1 . Then, the solitary wave solution of (1) is

$$
u(x, t)=3 \operatorname{csech}^{2}\left(k\left[x-x_{0}-v t\right]\right),
$$

where

$$
k=\frac{1}{2} \sqrt{\frac{c}{1+c}}, \quad v=1+c .
$$

We consider the motion of a single solitary wave and take as initial condition, with $c=0.1$ and $x_{0}=0$,

$$
u(x, 0)=0.3 \operatorname{sech}^{2}\left(\frac{x}{2 \sqrt{11}}\right) .
$$

The corresponding exact solution with initial condition (60) is

$$
u(x, t)=0.3 \operatorname{sech}^{2}\left(\frac{x-1.1 t}{2 \sqrt{11}}\right) .
$$

In this procedure, we take space-time domain as $-40 \leq$ $x \leq 60$ and $0 \leq t \leq 20$. 


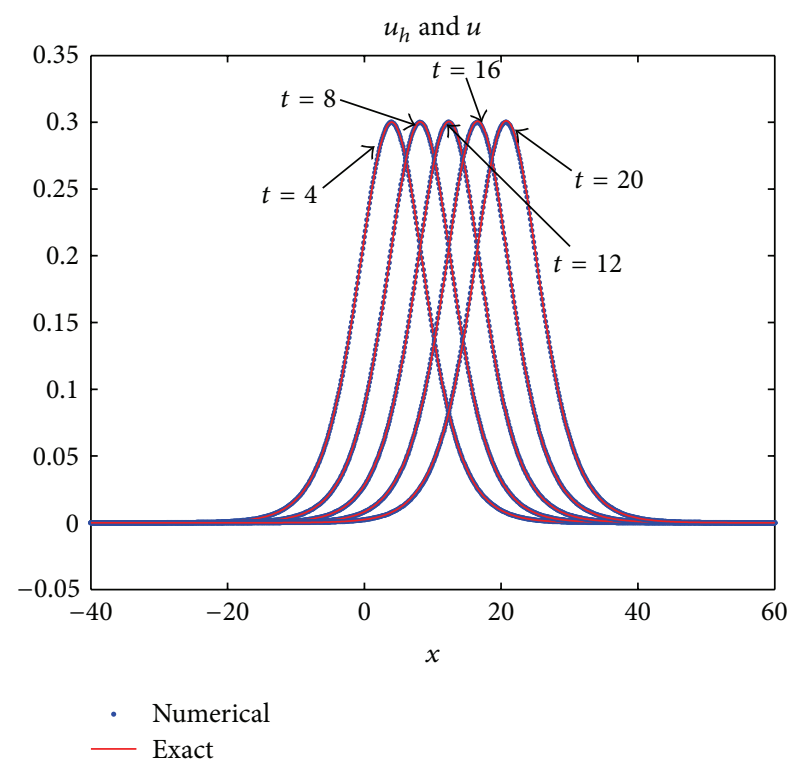

FIGURE 5: Comparison between $u$ and $u_{h}$ at times $t=4,8,12,16$, and 20 with $h=0.125$ and $\Delta t=0.2$.

In Table 1 , we take spatial mesh parameter $h=0.125$ and time discretization parameter $\Delta t=0.1$ and list the three invariants $Q_{1}, Q_{2}$, and $Q_{3}$ and the optimal error estimate in $L^{2}$ and $L^{\infty}$ norms for $u$ at different times $t=0,4,8,12,16$, and 20. At the same time, we show some numerical results at time $t=20$ obtained by other numerical methods in Table 1 . From Table 1, we find that our method is more accurate than the numerical methods in $[17,18,28]$ but is less than the numerical methods in $[23,24]$. From the shown results in Table 1 , we can see that $Q_{1}, Q_{2}$ and $Q_{3}$ keep almost constants, so the conservation for our method is very well.

In Tables 2 and 3, we take spatial mesh parameter $h=$ 0.125 and obtain the optimal error results in $L^{2}$ and $L^{\infty}$ norms for $u$ at different times $t=0,4,8,12,16$, and 20 with different time discretization parameters $\Delta t=0.1,0.2$, and 0.4 . From Tables 2 and 3, we see easily that the convergence rate for time is close to order 2. Similarly, the results for $q$ are shown in Tables 4 and 5.

In Tables 6 and 7, the optimal error results in $L^{2}$ and $L^{\infty}$ norms for $u$ at different times $t=0,4,8,12,16$, and 20 with different spatial mesh parameters $h=0.2,0.4$, and 0.8 and time discretization parameter $\Delta t=0.01$ are shown. It is easy to see that the convergence rate for space is close to order 2 . The similar results for $q$ are listed in Tables 8 and 9 .

Figure 1 shows the surface for the exact solution $u$ in space-time domain $((x, t) \in[-20,60] \times[0,20])$, and the corresponding surface for the numerical solution $u_{h}$ with $h=2$ and $\Delta t=0.4$ is described in Figure 2. From Figures 1 and 2 , we see easily that the exact solution $u$ is approximated very well by the numerical solution $u_{h}$. In Figures 3 and 4 , we show the surface for the exact solution $q$ and the numerical solution $q_{h}$, respectively, with $h=2$ and $\Delta t=0.4$ and obtain a good approximation solution $q_{h}$ for the exact solution $q$.

The comparison between the exact solution $u$ and the numerical solution $u_{h}$ is described at different times

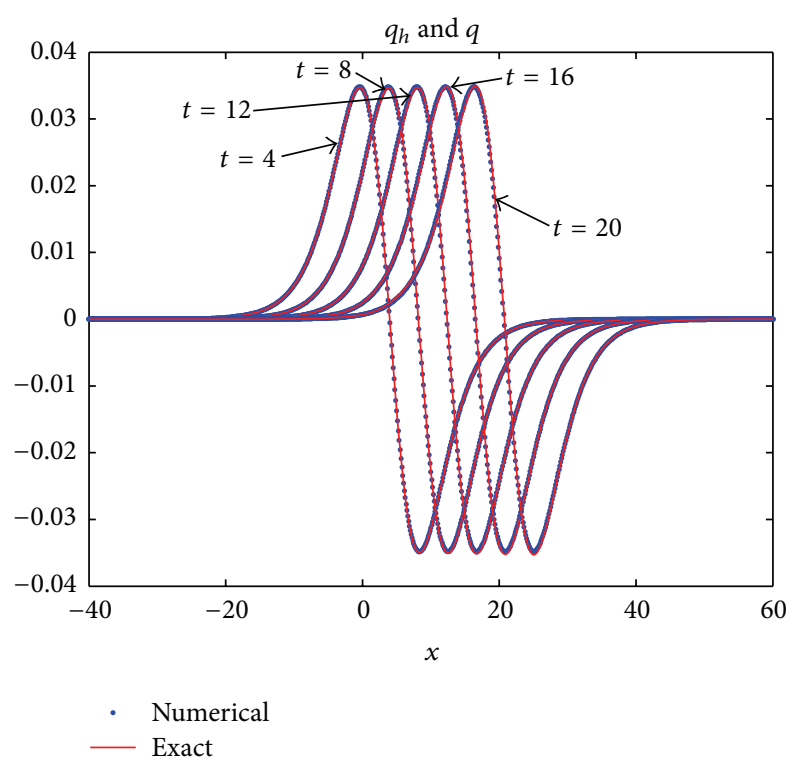

FIGURE 6: Comparison between $q$ and $q_{h}$ at times $t=48,12,16$, and 20 with $h=0.125$ and $\Delta t=0.2$.

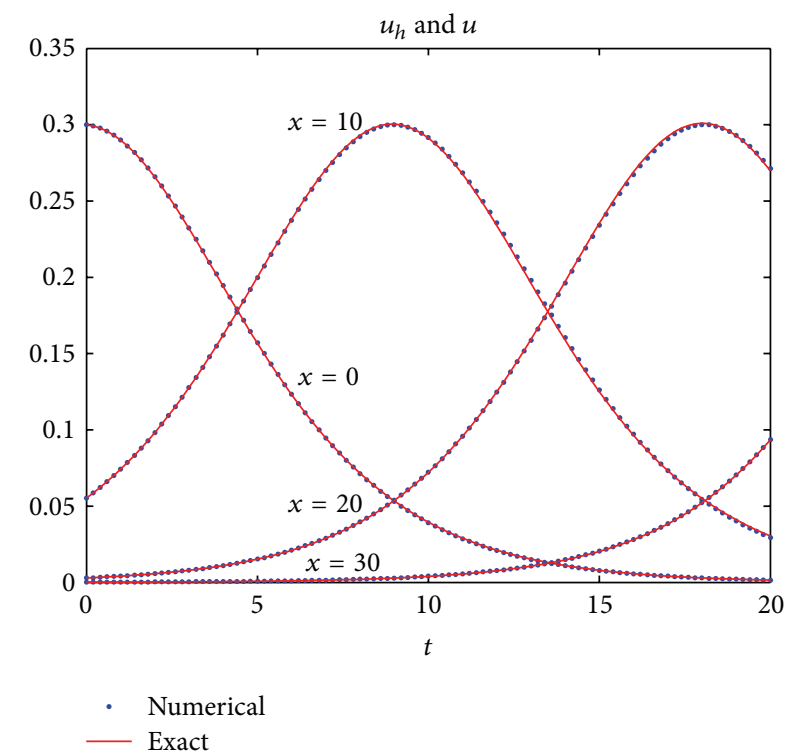

FIGURE 7: Comparison between $u$ and $u_{h}$ at spaces $x=0,10,20$, and 30 with $h=0.125$ and $\Delta t=0.2$.

$t=4,8,12,16$, and 20 with $h=0.125$ and $\Delta t=0.2$ in Figure 5. The similar comparison for the exact solution $q$ and the numerical solution $q_{h}$ is shown in Figure 6. Figures 5 and 6 show that the solitary wave for $u$ and $q$ moves to the right with unchanged form and velocity, respectively. Furthermore, the exact solutions $u$ and $q$ are approximated well by the numerical solutions $u_{h}$ and $q_{h}$, respectively.

In Figure 7, we show the comparison between $u$ and $u_{h}$ at different spaces $x=0,10,20$, and 30 with $h=0.125$ and $\Delta t=0.2$ to verify the efficiency for the proposed scheme in this paper. Figure 8 describes a similar result for $q$. 


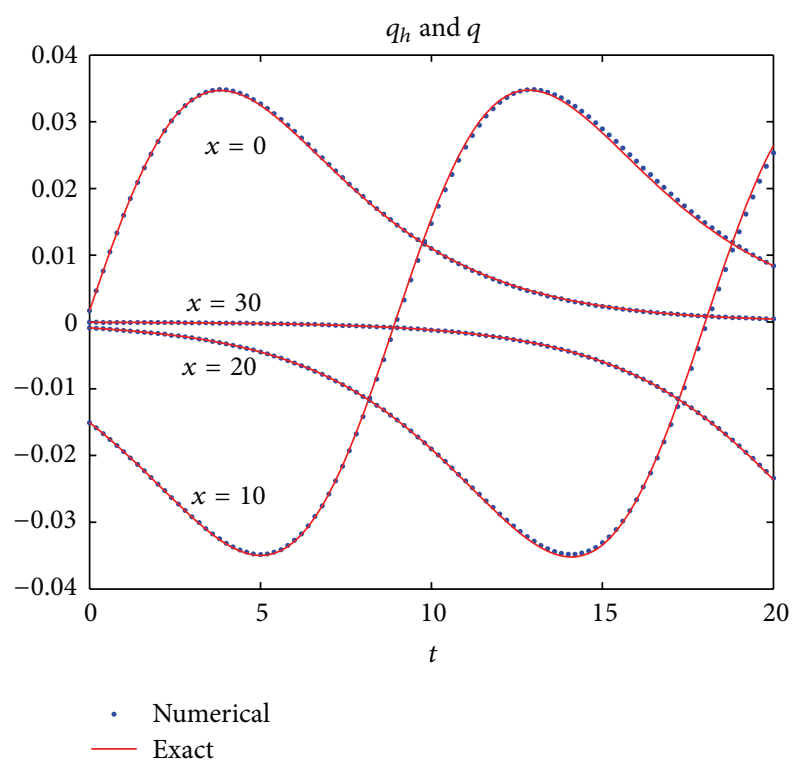

FIgURE 8: Comparison between $q$ and $q_{h}$ at spaces $x=0,10,20$, and 30 with $h=0.125$ and $\Delta t=0.2$.

From the previous analysis in Tables 1-9 and Figures 1-8, we can see that the numerical results confirm the theoretical results of Theorem 5 and our method is efficient for RLW equation.

\section{Concluding Remarks}

In this paper, we propose and analyze an explicit multistep mixed finite element method, which combines spatial mixed finite element method and time explicit multistep method, for RLW equation. We discuss the numerical process for our method, prove the theoretical results for the fully discrete explicit multistep mixed scheme, obtain the optimal convergence order, and compare our method's accuracy with some other numerical schemes. Compared with the numerical method in $[20,25,26]$, our method can obtain the optimal error estimates in $L^{2}$ and $H^{1}$ norms for the scalar unknown $u$ and its flux $q=u_{x}$ simultaneously. From our numerical results, we can see that our method is efficient for RLW equation.

\section{Acknowledgments}

This work was supported by the National Natural Science Fund of China (11061021), the Scientific Research Projection of Higher Schools of Inner Mongolia (NJZZ12011 and NJZY13199), the Natural Science Fund of Inner Mongolia Province (2012MS0108 and 2012MS0106), and the Program of Higher-Level Talents of Inner Mongolia University (125119).

\section{References}

[1] D. H. Peregrine, "Calculations of the development of an undular bore," Journal of Fluid Mechanics, vol. 25, no. 2, pp. 321-330, 1966.
[2] T. B. Benjamin, J. L. Bona, and J. J. Mahony, "Model equations for long waves in nonlinear dispersive systems," Philosophical Transactions of the Royal Society of London A, vol. 272, no. 1220, pp. 47-78, 1972.

[3] P. J. Olver, "Euler operators and conservation laws of the BBM equation," Mathematical Proceedings of the Cambridge Philosophical Society, vol. 85, no. 1, pp. 143-160, 1979.

[4] B. Saka and I. Dağ, "Quartic B-spline collocation algorithms for numerical solution of the RLW equation," Numerical Methods for Partial Differential Equations, vol. 23, no. 3, pp. 731-751, 2007.

[5] J. L. Bona and P. J. Bryant, "A mathematical model for long waves generated by wavemakers in non-linear dispersive systems," Mathematical Proceedings of the Cambridge Philosophical Society, vol. 73, no. 5, pp. 391-405, 1973.

[6] D. Bhardwaj and R. Shankar, "A computational method for regularized long wave equation," Computers \& Mathematics with Applications, vol. 40, no. 12, pp. 1397-1404, 2000.

[7] X. H. Zhao, D. S. Li, and D. M. Shi, "A finite difference scheme for RLW-Burgers equation," Journal of Applied Mathematics \& Informatics, vol. 26, no. 3-4, pp. 573-581, 2008.

[8] L. Zhang, "A finite difference scheme for generalized regularized long-wave equation," Applied Mathematics and Computation, vol. 168, no. 2, pp. 962-972, 2005.

[9] I. Dağ, A. Dogan, and B. Saka, "B-spline collocation methods for numerical solutions of the RLW equation," International Journal of Computer Mathematics, vol. 80, no. 6, pp. 743-757, 2003.

[10] A. Esen and S. Kutluay, "Application of a lumped Galerkin method to the regularized long wave equation," Applied Mathematics and Computation, vol. 174, no. 2, pp. 833-845, 2006.

[11] I. Dağ, B. Saka, and D. Irk, "Galerkin method for the numerical solution of the RLW equation using quintic B-splines," Journal of Computational and Applied Mathematics, vol. 190, no. 1-2, pp. 532-547, 2006.

[12] K. R. Raslan, "A computational method for the regularized long wave (RLW) equation," Applied Mathematics and Computation, vol. 167, no. 2, pp. 1101-1118, 2005.

[13] L. Wahlbin, "Error estimates for a Galerkin method for a class of model equations for long waves," Numerische Mathematik, vol. 23, pp. 289-303, 1975.

[14] Z. D. Luo and R. X. Liu, "Mixed finite element analysis and numerical solitary solution for the RLW equation," SIAM Journal on Numerical Analysis, vol. 36, no. 1, pp. 89-104, 1999.

[15] L. Guo and H. Chen, " $H^{1}$-Galerkin mixed finite element method for the regularized long wave equation," Computing, vol. 77, no. 2, pp. 205-221, 2006.

[16] H. M. Gu and N. Chen, "Least-squares mixed finite element methods for the RLW equations," Numerical Methods for Partial Differential Equations, vol. 24, no. 3, pp. 749-758, 2008.

[17] J. L. Bona, W. G. Pritchard, and L. R. Scott, "Numerical schemes for a model for nonlinear dispersive waves," Journal of Computational Physics, vol. 60, no. 2, pp. 167-186, 1985.

[18] S. U. Islam, S. Haq, and A. Ali, "A meshfree method for the numerical solution of the RLW equation," Journal of Computational and Applied Mathematics, vol. 223, no. 2, pp. 997-1012, 2009.

[19] D. Kaya, "A numerical simulation of solitary-wave solutions of the generalized regularized long-wave equation," Applied Mathematics and Computation, vol. 149, no. 3, pp. 833-841, 2004.

[20] L. Q. Mei and Y. P. Chen, "Explicit multistep method for the numerical solution of RLW equation," Applied Mathematics and Computation, vol. 218, no. 18, pp. 9547-9554, 2012. 
[21] L. R. T. Gardner, G. A. Gardner, and A. Dogan, "A least-squares finite element scheme for the RLW equation," Communications in Numerical Methods in Engineering, vol. 12, no. 11, pp. 795-804, 1996.

[22] I. Dağ, "Least squares quadratic B-spline finite element method for the regularized long wave equation," Computer Methods in Applied Mechanics and Engineering, vol. 182, no. 1-2, pp. 205215, 2000.

[23] I. Dağ and M. N. Özer, "Approximation of the RLW equation by the least square cubic B-spline finite element method," Applied Mathematical Modelling, vol. 25, no. 3, pp. 221-231, 2001.

[24] A. Dogan, "Numerical solution of RLW equation using linear finite elements within Galerkin's method," Applied Mathematical Modelling, vol. 26, no. 7, pp. 771-783, 2002.

[25] P. Chatzipantelidis, "Explicit multistep methods for nonstiff partial differential equations," Applied Numerical Mathematics, vol. 27, no. 1, pp. 13-31, 1998.

[26] G. Akrivis, O. Karakashian, and F. Karakatsani, "Linearly implicit methods for nonlinear evolution equations," Numerische Mathematik, vol. 94, no. 3, pp. 403-418, 2003.

[27] A. K. Pani, R. K. Sinha, and A. K. Otta, "An $H^{1}$-Galerkin mixed method for second order hyperbolic equations," International Journal of Numerical Analysis and Modeling, vol. 1, no. 2, pp. 111129, 2004.

[28] M. F. Wheeler, "A priori $L_{2}$ error estimates for Galerkin approximations to parabolic partial differential equations," SIAM Journal on Numerical Analysis, vol. 10, pp. 723-759, 1973.

[29] J. L. Bona, W. G. Pritchard, and L. R. Scott, "An evaluation of a model equation for water waves," Philosophical Transactions of the Royal Society of London A, vol. 302, no. 1471, pp. 457-510, 1981. 


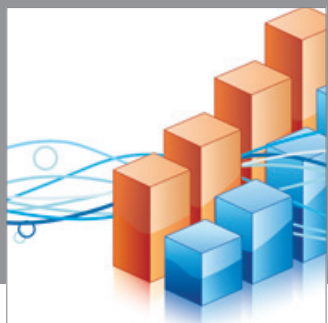

Advances in

Operations Research

mansans

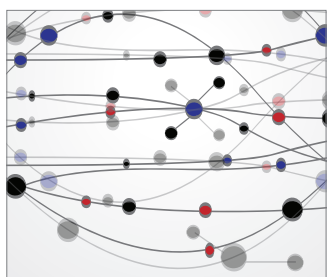

The Scientific World Journal
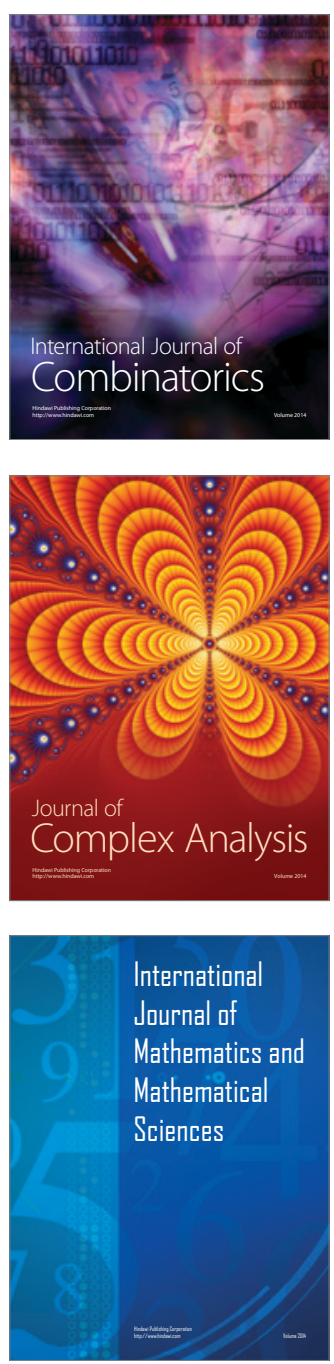
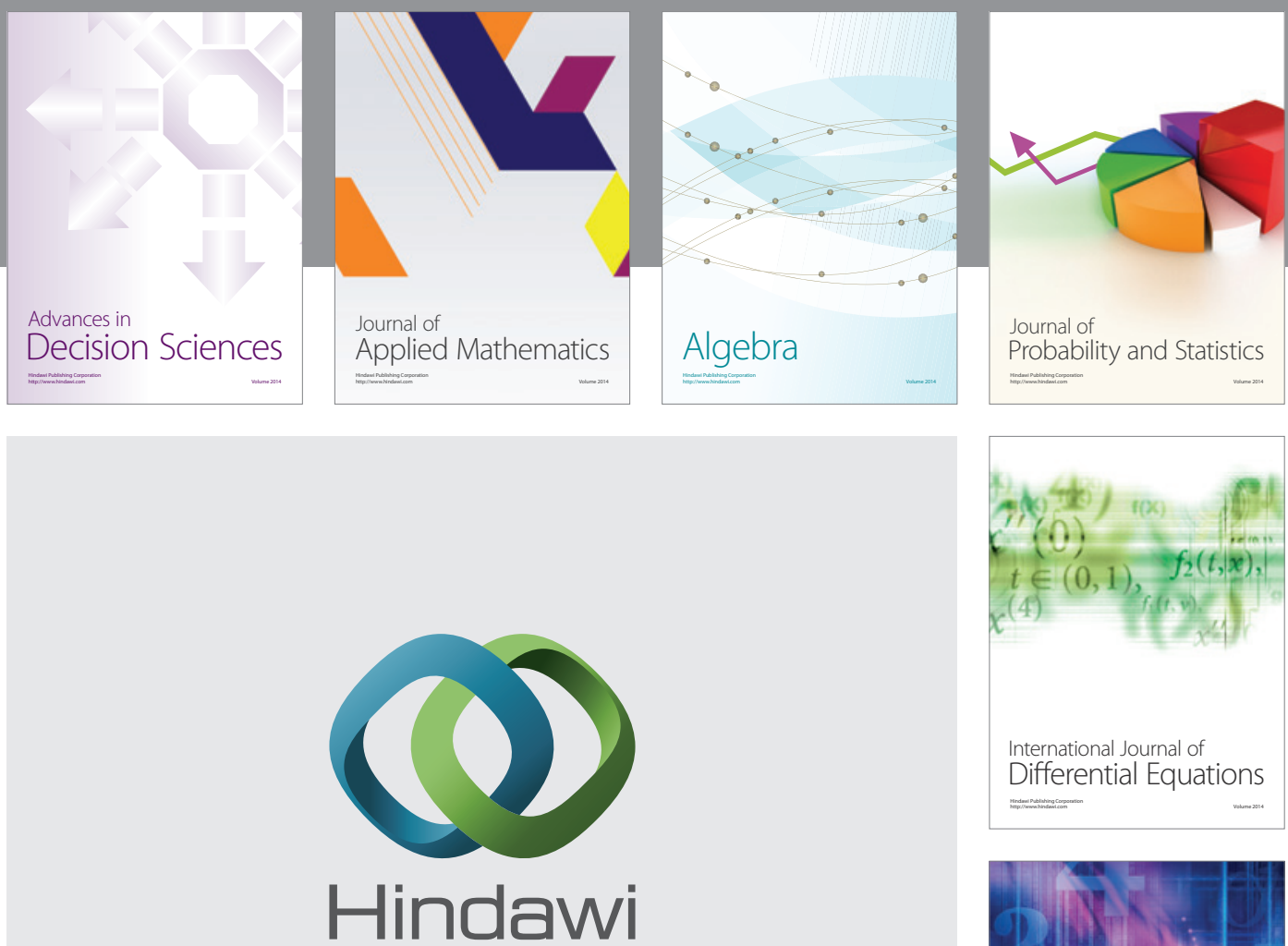

Submit your manuscripts at http://www.hindawi.com
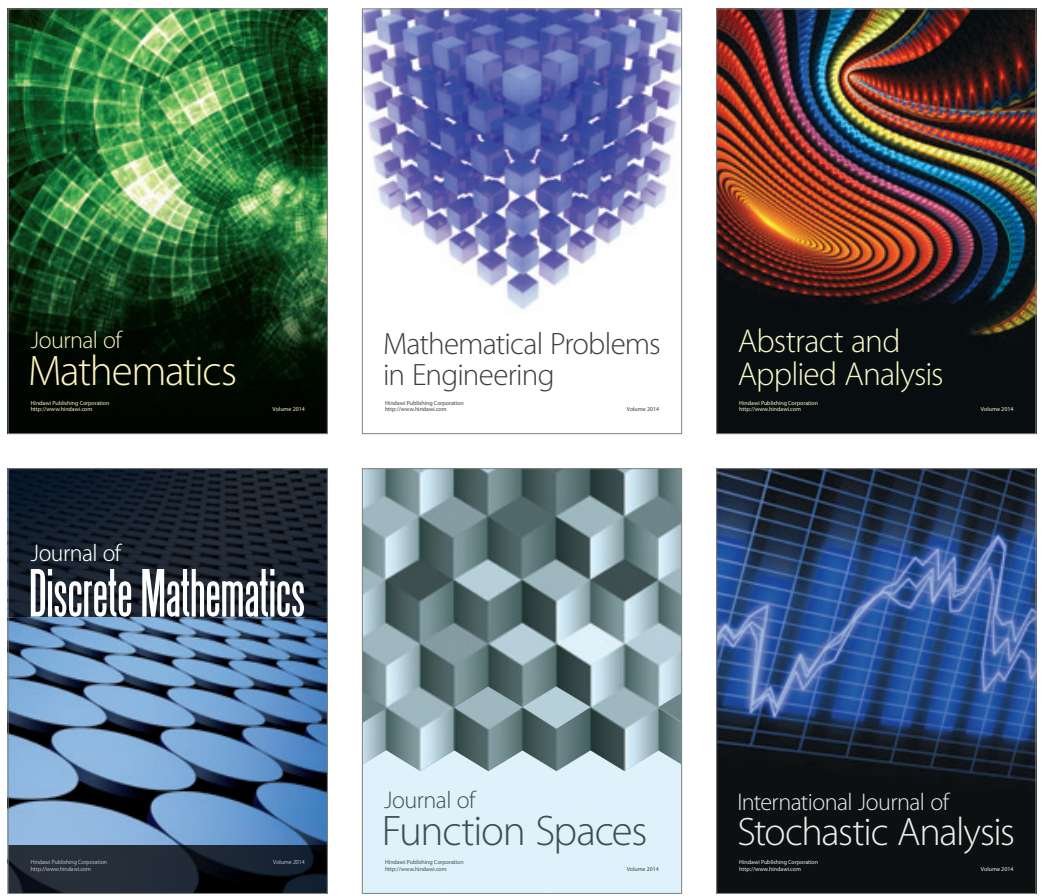

Journal of

Function Spaces

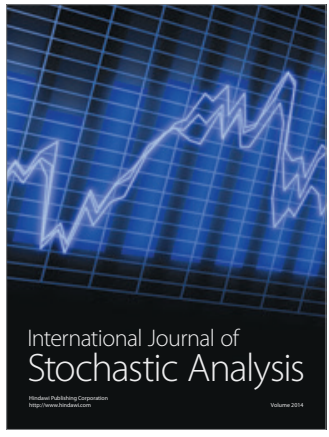

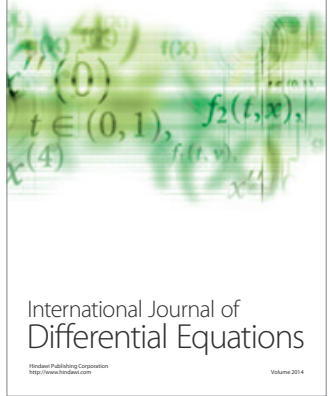
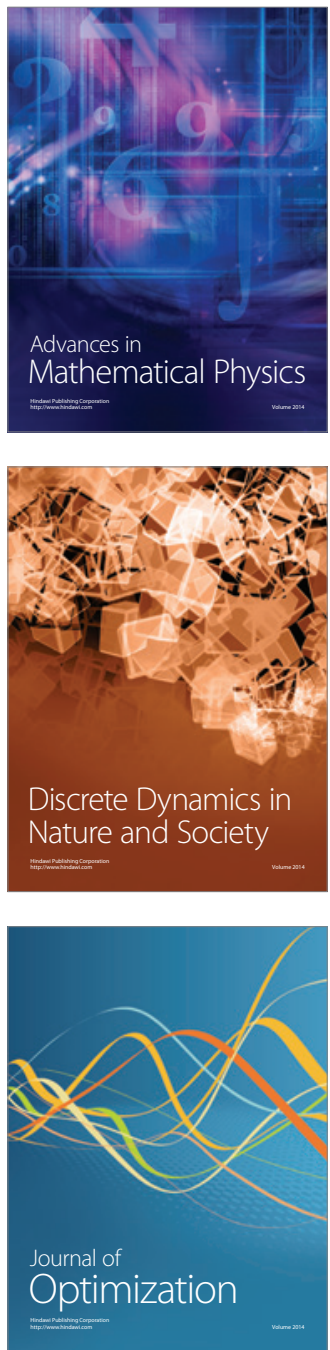